\section{RSP}

http://www.rsp.fsp.usp.br/
Revista de Saúde Pública

\title{
Consumo de hortaliças e sua relação com os alimentos ultraprocessados no Brasil
}

\author{
Daniela Silva Canella',II, Maria Laura da Costa Louzada ${ }^{\prime \prime, I I I,}$ Rafael Moreira Claro ${ }^{\prime \prime, I V}$, Janaina Calu \\ Costav, Daniel Henrique Bandoni" ${ }^{\mathrm{VI}}$, Renata Bertazzi Levy ${ }^{\mathrm{II}, \mathrm{VII}}$, Ana Paula Bortoletto Martins"
}

' Universidade do Estado do Rio de Janeiro. Instituto de Nutrição. Departamento de Nutrição Aplicada. Rio de Janeiro, RJ, Brasil

" Universidade de São Paulo. Núcleo de Pesquisas Epidemiológicas em Nutrição e Saúde. São Paulo, SP, Brasil

III Universidade Federal de São Paulo. Instituto de Saúde e Sociedade. Departamento de Políticas Públicas e Saúde Coletiva. Santos, SP, Brasil

iv Universidade Federal de Minas Gerais. Escola de Enfermagem. Departamento de Nutrição. Belo Horizonte, MG, Brasil

$\checkmark$ Universidade Federal de Pelotas. Programa de Pós-Graduação em Epidemiologia. Pelotas, RS, Brasil

vı Universidade Federal de São Paulo. Instituto de Saúde e Sociedade. Departamento de Saúde, Clínica e Instituições. Santos, SP, Brasil

VII Universidade de São Paulo. Faculdade de Medicina. Departamento de Medicina Preventiva. São Paulo, SP, Brasil

\section{RESUMO}

OBJETIVO: Caracterizar a aquisição domiciliar e o consumo alimentar individual de hortaliças no Brasil e analisar sua relação com o consumo de alimentos ultraprocessados.

MÉTODOS: Foram utilizados dados de aquisição de alimentos para consumo no domicílio e de consumo alimentar individual da Pesquisa de Orçamentos Familiares 2008-2009. A Pesquisa de Orçamentos Familiares estudou a aquisição de alimentos de 55.970 domicílios e o consumo alimentar de 34.003 indivíduos com 10 anos ou mais de idade. Os alimentos de interesse neste estudo foram as hortaliças (excluindo raízes e tubérculos) e os alimentos ultraprocessados. A quantidade de hortaliças (gramas) adquiridas e consumidas foi descrita para o conjunto dos brasileiros e segundo quintos da participação calórica de alimentos ultraprocessados na alimentação. Para tanto, foram calculados os valores brutos e preditos, obtidos por modelos de regressão ajustados por variáveis sociodemográficas. Analisaram-se os tipos mais adquiridos de hortaliças (\% na quantidade total) e, em relação ao consumo alimentar individual, a variedade de hortaliças consumidas (número absoluto), a participação (\%) dos tipos de preparação culinária à base de hortaliças e os horários de consumo.

RESULTADOS: A aquisição domiciliar média ajustada de hortaliças foi $42,9 \mathrm{~g} /$ per capita/dia. O consumo individual médio ajustado foi $46,1 \mathrm{~g}$. Verificou-se relação inversa entre aquisição domiciliar e consumo individual de hortaliças e de alimentos ultraprocessados. Dez tipos de hortaliças respondem por mais de $80 \%$ da quantidade total habitualmente adquirida. A variedade consumida foi, em média, 1,08 tipo/per capita/dia. Cerca de 60\% das hortaliças foram consumidas cruas, sendo a quantidade consumida no almoço duas vezes maior que aquela do jantar e indivíduos com maior consumo de alimentos ultraprocessados tenderam a consumir quantidade ainda menor de hortaliças no jantar.

CONCLUSÕES: O consumo de hortaliças no Brasil é insuficiente, sendo pior entre indivíduos com maior consumo de alimentos ultraprocessados. O hábito mais frequente foi consumir hortaliças cruas, no almoço e com limitada variedade.

DESCRITORES: Alimentos Industrializados. Verduras, economia. Consumo de Alimentos. Comportamento Alimentar. Inquéritos sobre Dietas. Alimentos, Dieta e Nutrição. 


\section{INTRODUÇÃO}

Frutas e hortaliças são importantes fontes de vitaminas, minerais, fibras, e outros compostos bioativos, além de apresentarem baixa densidade energética, fazendo de seu consumo em níveis adequados um importante fator protetor para morbidade (doenças cardiovasculares, hipertensão, diabetes e alguns tipos de câncer) e mortalidade ${ }^{1-3}$. Entretanto, apesar das recomendações internacionais e nacionais de consumo e da variedade de tipos e de preparações culinárias em que esses alimentos podem ser ingeridos ${ }^{1,4,5, a}$, seu consumo permanece aquém do desejado em grande parte dos países, sendo o cenário ainda pior para as hortaliças quando comparado ao de frutas ${ }^{6-8}$.

No Brasil, entre 1987-1988 e 2008-2009, observou-se certa estabilidade e baixos níveis de aquisição de frutas e hortaliças, inclusive com pequena retração no consumo das hortaliças. Em 2008, a participação combinada desses alimentos na alimentação representou 2,9\% do total de calorias, sendo $2,2 \%$ para as frutas e $0,7 \%$ para as hortaliças. No entanto, nesse mesmo período, houve acentuado declínio da participação de alimentos in natura na alimentação da população brasileira concomitantemente ao aumento expressivo no consumo de alimentos ultraprocessados, acarretando redução da qualidade global da alimentação no país .

Em relação ao consumo especificamente de hortaliças, apenas 35\% dos adolescentes e $38 \%$ dos adultos (residentes nas capitais de Estado e Distrito Federal) referiram consumo regular (em cinco ou mais dias da semana), enquanto, respectivamente, $20 \%$ e $8 \%$ referiram não consumir ${ }^{b, c}$. Para fins de comparação, entre os adolescentes brasileiros, a frequência de consumo regular observada para as hortaliças no ano de 2012 já era inferior àquela encontrada para as guloseimas $(41,3 \%)$ e muito semelhante àquelas observadas para os biscoitos doces $(32,5 \%)$ e salgados $(35,1 \%)$ e para os refrigerantes $(33,2 \%)^{10}$.

Diante desse cenário, o Guia Alimentar para a População Brasileira recomenda a substituição do consumo de alimentos ultraprocessados por alimentos in natura ou minimamente processados. Essa estratégia visa à melhoria efetiva na qualidade da alimentação ${ }^{11,12}$, valorizando a variedade de alimentos de origem vegetal disponível, respeitando a diversidade biossociocultural e alimentar do país e explorando as possibilidades de combinações e preparações culinárias ${ }^{1}$.

${ }^{a}$ Ministério da Saúde (BR), Secretaria de Atenção à Saúde, Departamento de Atenção Básica. Alimentos regionais brasileiros. 2.ed. Brasília (DF); 2015 [citado 2 mar 2017]. Disponível em: http://bvsms. saude.gov.br/bvs/publicacoes/ alimentos_regionais_brasileiros

${ }^{b}$ Ministério da Saúde (BR), Secretaria de Vigilância em Saúde, Departamento de Vigilância de Doenças e Agravos não Transmissíveis e Promoção da Saúde. Vigitel Brasil 2014: vigilância de fatores de risco e proteção para doenças crônicas por inquérito telefônico. Brasília (DF); 2015 [citado 2 mar 2017]. Disponível em: http://bvsms. saude.gov.br/bvs/publicacoes/ vigitel_brasil_2014.pdf

c Instituto Brasileiro de Geografia e Estatística. Pesquisa Nacional de Saúde do Escolar 2012. Rio de Janeiro: IBGE; 2013.

d Instituto Brasileiro de Geografia e Estatística. Pesquisa de Orçamentos Familiares 20082009: análise da disponibilidade domiciliar de alimentos e do estado nutricional no Brasil. Rio de Janeiro: IBGE; 2010.

Dado o contexto do consumo de hortaliças e de alimentos ultraprocessados no país, as recomendações do Guia Alimentar para a População Brasileira e a escassez de estudos que explorem o consumo de hortaliças para além da adequação em quantidade e da regularidade, este estudo teve como objetivo caracterizar o consumo de hortaliças no Brasil e analisar sua relação com o consumo de alimentos ultraprocessados.

\section{MÉTODOS}

\section{Fonte de Dados, Amostra e Coleta de Dados}

Foram utilizados dados da Pesquisa de Orçamentos Familiares (POF) realizada pelo Instituto Brasileiro de Geografia e Estatística (IBGE) entre maio de 2008 e maio de 2009. Trata-se de pesquisa com dados representativos da população brasileira, distribuída nas cinco macrorregiões e nas áreas urbana e rural do país.

A POF 2008-2009 utilizou plano amostral complexo, por conglomerados, envolvendo estratificação geográfica e socioeconômica de todos os setores censitários do país, seguida de sorteio aleatório de setores no primeiro estágio, e de domicílios no segundo estágio. No total, 68.373 domicílios foram selecionados, dos quais 55.970 foram efetivamente entrevistados. A coleta de dados foi realizada ao longo de 12 meses, de maneira uniforme nos estratos, garantindo a representatividade nos quatro trimestres do ano ${ }^{\mathrm{d}}$.

No presente estudo, foram utilizados dados referentes à aquisição domiciliar de alimentos, registrada por todos os domicílios amostrados, e ao consumo alimentar individual, coletado 
em subamostra aleatória da população (13.569 domicílios, equivalente a 24,3\% do total, envolvendo todos os indivíduos com mais de 10 anos, totalizando 34.003 indivíduos).

Foram utilizados também dados referentes à renda domiciliar per capita $(\mathrm{R} \$ /$ per capita/ mês), área (urbana ou rural) e região em que o domicílio se localiza (Norte, Nordeste, CentroOeste, Sudeste e Sul), a data de nascimento (utilizada no cálculo da idade e estimada em anos completos) e sexo dos indivíduos estudados.

\section{Aquisição de Alimentos para Consumo no Domicílio}

Os dados de aquisição de alimentos para consumo no domicílio foram obtidos por meio do registro de alimentos e bebidas adquiridos, anotados em caderneta específica pelos próprios moradores dos domicílios (ou entrevistador, quando necessário) durante sete dias consecutivos ${ }^{\text {d }}$. Devido ao curto período de referência empregado para o registro das despesas com alimentação em cada domicílio, optou-se por utilizar, como unidade de estudo, agregados de domicílios, definidos com base nos 550 estratos amostrais, cujo padrão de aquisição anual de alimentos pode ser conhecido com maior precisão. O número médio de domicílios estudados dentro de cada estrato foi de 101,8, variando entre oito e 796 domicílios.

A quantidade semanal adquirida de cada item alimentar, após excluída sua fração não comestível, foi dividida por sete (número de dias na semana) a fim de expressar valores diários de consumo. Em seguida, para cada alimento, a quantidade líquida diária adquirida, em gramas ou mililitros, foi convertida em energia, empregando-se a Tabela Brasileira de Composição de Alimentos (TACO) ${ }^{\mathrm{e}}$ ou, quando o alimento não estivesse presente nessa tabela, a tabela de composição de alimentos do Departamento de Agricultura dos Estados Unidos (United States Department of Agriculture - USDA) versão $23^{\mathrm{f}}$.

\section{Consumo Alimentar Individual}

Os dados de consumo alimentar foram obtidos por meio de dois registros diários da alimentação em dias não consecutivos. Os indivíduos com 10 anos ou mais residentes nos domicílios amostrados registraram todos os alimentos e bebidas que consumiram em um período de 24 horas, indicando as quantidades consumidas em medidas caseiras e a forma de preparação . Para este estudo, foram considerados apenas os dados referentes ao consumo de indivíduos que preencheram os dois dias de registro alimentar $(\mathrm{n}=32.900)$.

Campinas, Núcleo de Estudos e Pesquisas em Alimentação.

Tabela Brasileira de Composição de Alimentos - TACO. Campinas: UNICAMP; 2004.

${ }^{\mathrm{f}}$ United States, Department of Agriculture. USDA National Nutrient Database for Standard Reference. Release 23.

Beltsville (MD): Nutrient Data Laboratory; 2010.

${ }^{8}$ Instituto Brasileiro de Geografia e Estatística. Análise do consumo alimentar pessoal no Brasil. Instituto Brasileiro de Geografia e Estatística. Rio de Janeiro; 2011.

${ }^{\mathrm{h}}$ Instituto Brasileiro de Geografia e Estatística. Tabela de medidas referidas para os alimentos consumidos no Brasil. Rio de Janeiro: IBGE; 2011.

`Instituto Brasileiro de Geografia e Estatística. Tabelas de composição nutricional dos alimentos consumidos no Brasil. Rio de Janeiro: IBGE; 2011.

j Bombem KCM, Canella DS Bandoni DH, Jaime PC. Manual de medidas caseiras e receitas para cálculos dietéticos. São Paulo: M. Books do Brasil; 2012.

A quantidade de cada alimento ou bebida, informada pelo indivíduo em medidas caseiras, foi transformada em gramas ou mililitros utilizando a Tabela de Medidas Referidas para os Alimentos Consumidos no Brasil h . A seguir, as quantidades de alimentos ou bebidas foram convertidas em energia com base na Tabela de Composição Nutricional dos Alimentos Consumidos no Brasili.

\section{Definição dos Indicadores do Estudo}

Os alimentos de interesse neste estudo foram as hortaliças (incluindo legumes e verduras, em todas as formas de preparo, e excluindo raízes e tubérculos) e os alimentos ultraprocessados.

Quanto às hortaliças, em relação à aquisição para consumo domiciliar, considerou-se todas as hortaliças adquiridas. No caso dos dados de consumo alimentar individual, além da inclusão das hortaliças consumidas (i) cruas (por exemplo, em forma de saladas) e (ii) cozidas, optou-se por incluir as preparações culinárias à base de hortaliças, divididas entre: (iii) refogadas, alho e óleo ou na manteiga; (iv) com molho ou ensopadas; (v) fritas, empanadas ou à milanesa; (vi) grelhadas, na brasa, assadas ou churrasco; e (vii) sopas. No caso dessas cinco preparações culinárias, em função da inclusão de outros ingredientes que afetam o peso final da preparação, foi empregada padronização da participação de hortaliças para cada tipo de preparação com o intuito de estimar a quantidade de hortaliças consumidas. No caso das sopas, dada a quantidade de líquido e de outros ingredientes, considerou-se que as hortaliças representavam $25 \%$ do peso total da preparaçãoj. Para as demais preparações, considerou-se o percentual adotado na própria POFi. 
Já o grupo de alimentos ultraprocessados foi definido com base na classificação NOVA ${ }^{13}$, a saber: alimentos ultraprocessados são formulações industriais prontas para consumo e feitas inteiramente ou majoritariamente de substâncias extraídas de alimentos (óleos, gorduras, açúcar, amido, proteínas), derivadas de constituintes de alimentos (gorduras hidrogenadas, amido modificado) ou sintetizadas em laboratório com base em matérias orgânicas (corantes, aromatizantes, realçadores de sabor e vários tipos de aditivos usados para dotar os produtos de propriedades sensoriais atraentes). Esse grupo inclui os seguintes subgrupos de alimentos: bolachas salgadas e salgadinhos tipo chips; bolos, tortas e biscoitos doces; cereais matinais; embutidos, hambúrgueres e salsichas; guloseimas (balas, confeitos, chocolates, gelatina, pudins e sorvetes); lanches do tipo fast food (hambúrguer e cheeseburguer, hot dog, salgados fritos e assados e semelhantes); margarina; molhos industrializados; pães de forma, de hambúrguer, de hot dog e similares; pratos prontos ou semiprontos (pizzas, pratos de massa ou de carne congelados, macarrão instantâneo e sopas em pó); queijos ultraprocessados; refrigerantes e outras bebidas açucaradas.

\section{Análise dos Dados}

Inicialmente, as quantidades de hortaliças (grama/per capita/dia) e de alimentos ultraprocessados (kcal/per capita/dia) adquiridas para consumo no domicílio e consumidas pelos indivíduos foram descritas para o Brasil e segundo características sociodemográficas.

As quantidades (grama/per capita/dia) de hortaliças - adquiridas pelos domicílios e consumidas pelos indivíduos - foram descritas para o conjunto da população brasileira e segundo quintos da participação calórica de alimentos ultraprocessados na alimentação. Em seguida, foram calculados, por modelos de regressão linear múltipla, os valores preditos (ajustados) para a aquisição domiciliar e consumo individual de hortaliças levando em conta as características sociodemográficas. No caso da aquisição para consumo domiciliar de hortaliças, consideramos a renda per capita (em logaritmo natural), a área e a região; e para o consumo individual de hortaliças, a renda per capita (em logaritmo natural), a área, a região, o sexo e a idade.

Procedimento de ajuste análogo foi adotado para descrição da participação dos 10 tipos de hortaliças mais adquiridos para consumo domiciliar no total de hortaliças adquiridas no Brasil e nos grupos com menor ( $1^{\circ}$ quinto) e maior ( $5^{\circ}$ quinto) participação de alimentos ultraprocessados na alimentação. A diferença na participação de cada tipo de hortaliças entre os grupos de consumo de alimentos ultraprocessados ( $1^{\circ}$ e $5^{\circ}$ quintos) foi testada por modelos de regressão linear múltipla.

Em relação ao consumo alimentar individual de hortaliças, analisou-se a variedade de hortaliças consumidas (expresso em número absoluto), a participação (\%) dos tipos de preparações culinárias à base de hortaliças no consumo total de hortaliças e os horários de consumo. A variedade ajustada de hortaliças consumidas por indivíduo e a participação ajustada dos tipos de preparações no consumo foram descritas para o conjunto de brasileiros estudados e para os brasileiros com menor ( $1^{\circ}$ quinto) e maior ( $5^{\circ}$ quinto) consumo de alimentos ultraprocessados. Ainda, foi descrita a quantidade ajustada de hortaliças consumidas (em grama), segundo horário de consumo, também para o conjunto de brasileiros estudados e para aqueles com menor e maior consumo de alimentos ultraprocessados. A diferença entre os grupos de consumo de alimentos ultraprocessados ( $1^{\circ}$ e $5^{\circ}$ quintos) para variedade, cada tipo de preparação e cada horário foi testada por modelos de regressão linear múltipla.

Todas as análises foram realizadas com o emprego do software Stata (StataCorp LP, College Station, Texas, United States), versão 14.2, levando-se em conta o delineamento complexo da amostra. 


\section{RESULTADOS}

A quantidade média de hortaliças adquiridas para consumo nos domicílios brasileiros foi de 43,7 grama/per capita/dia e o consumo individual diário médio, de 49,2 grama/per capita/ dia. Em relação aos alimentos ultraprocessados, a aquisição domiciliar média representou 18,0\% das calorias totais e o consumo individual, 20,5\% (Tabela 1).

A quantidade de hortaliças e de alimentos ultraprocessados referente à aquisição domiciliar aumenta com a elevação da renda e difere entre as regiões: para as hortaliças, foi maior nas regiões Sul, Sudeste e Centro-Oeste e para os alimentos ultraprocessados, nas regiões Sul e Sudeste. Para os alimentos ultraprocessados, ainda se observa que a quantidade adquirida na área urbana é maior. Em relação ao consumo alimentar individual de hortaliças e de alimentos ultraprocessados, observou-se resultados similares para renda, áreas e regiões, e a única diferença, em relação à aquisição, foi o maior consumo de hortaliças na área urbana. Ademais, mulheres consomem maior quantidade de alimentos ultraprocessados, e a idade apresentou relação inversa com o consumo de alimentos ultraprocessados e relação direta com o consumo de hortaliças (Tabela 1).

A quantidade média de hortaliças adquiridas para consumo nos domicílios brasileiros, após ajuste para variáveis sociodemográficas, foi de $42,9 \mathrm{~g} /$ per capita/dia. Observou-se, nas análises ajustadas, relação inversa entre a aquisição de hortaliças e de alimentos ultraprocessados, ou seja, domicílios com maior participação calórica de alimentos ultraprocessados na alimentação adquiriram menor quantidade de hortaliças. No tocante aos dados de consumo alimentar individual, após ajuste para variáveis sociodemográficas, verificou-se um valor médio de 46,1 g/per capita/dia. Também foi verificada relação inversa entre o consumo de hortaliças e de alimentos ultraprocessados (Tabela 2).

Dez tipos de hortaliças representaram 83\% da quantidade (em gramas) habitualmente adquirida pelos brasileiros para consumo no domicílio, não sendo essa variedade afetada pela participação de alimentos ultraprocessados na alimentação $\left(82,7 \%\right.$ no caso do $1^{\circ}$

Tabela 1. Quantidade de hortaliças (em grama) e alimentos ultraprocessados (em kcal) adquiridos e consumidos no Brasil, segundo características sociodemográficas. Brasil, 2008-2009.

\begin{tabular}{|c|c|c|c|c|}
\hline \multirow[b]{2}{*}{ Variável } & \multicolumn{2}{|c|}{$\begin{array}{l}\text { Aquisição domiciliar de alimentos } \\
\text { ( } \mathrm{n}=55.970 \text { domicílios })\end{array}$} & \multicolumn{2}{|c|}{$\begin{array}{l}\text { Consumo alimentar individual } \\
(\mathrm{n}=32.900 \text { indivíduos })\end{array}$} \\
\hline & $\begin{array}{c}\text { Hortaliças } \\
\text { (g/per capita/dia) } \\
\text { (IC95\%) }\end{array}$ & $\begin{array}{c}\text { Alimentos } \\
\text { ultraprocessados } \\
\text { (kcal/per capita/dia) } \\
(\text { IC95\%) }\end{array}$ & $\begin{array}{c}\text { Hortaliças } \\
\text { (g/per capita/dia) } \\
\text { (IC95\%) }\end{array}$ & $\begin{array}{c}\text { Alimentos } \\
\text { ultraprocessados } \\
\text { (kcal/per capita/dia) } \\
(\text { IC95\%) }\end{array}$ \\
\hline \multicolumn{5}{|c|}{ Renda domiciliar per capita $(\mathrm{R} \$)$} \\
\hline $1^{\circ}$ terço & $33,0(31,0-34,9)$ & $12,4(11,7-13,2)$ & $31,5(30,2-32,8)$ & $15,1(14,6-15,5)$ \\
\hline $2^{\circ}$ terço & $42,1(37,8-46,2)$ & $17,6(16,5-18,8)$ & $48,3(46,7-50,0)$ & $20,2(19,7-20,8)$ \\
\hline $3^{\circ}$ terço & $56,1(52,0-60,1)$ & $23,9(22,8-24,9)$ & $67,6(64,9-70,3)$ & $26,3(25,7-26,9)$ \\
\hline \multicolumn{5}{|l|}{ Área } \\
\hline Rural & $39,2(35,3-43,0)$ & $10,6(9,7-11,4)$ & $41,3(39,5-43,2)$ & $12,7(12,3-13,2)$ \\
\hline Urbana & $44,5(41,8-47,2)$ & $19,3(18,5-20,2)$ & $50,7(49,4-52,1)$ & $22,1(21,7-22,5)$ \\
\hline \multicolumn{5}{|l|}{ Região } \\
\hline Norte & $31,9(28,6-35,3)$ & $11,7(10,4-13,0)$ & $28,8(27,0-30,5)$ & $14,8(14,3-15,4)$ \\
\hline Nordeste & $37,2(34,4-40,0)$ & $14,6(13,7-15,4)$ & $30,5(29,2-31,8)$ & $14,9(14,5-15,3)$ \\
\hline Sudeste & $45,6(40,9-50,2)$ & $20,2(18,9-21,5)$ & $56,4(54,4-58,5)$ & $23,6(23,0-24,2)$ \\
\hline Sul & $53,4(49,4-57,4)$ & $22,0(20,6-23,3)$ & $60,0(60,1-68,0)$ & $25,7(25,0-26,4)$ \\
\hline Centro-Oeste & $45,9(40,8-51,0)$ & $14,5(13,2-15,8)$ & $67,5(64,1-70,8)$ & $19,4(18,4-20,3)$ \\
\hline \multicolumn{5}{|l|}{ Sexo } \\
\hline Masculino & - & - & $47,6(45,9-49,2)$ & $19,2(18,7-19,7)$ \\
\hline Feminino & - & - & $50,6(49,1-52,3)$ & $21,8(21,3-22,2)$ \\
\hline \multicolumn{5}{|l|}{ Idade (anos) } \\
\hline 10-19 & - & - & $31,6(29,9-33,3)$ & $26,8(26,1-27,6)$ \\
\hline $20-39$ & - & - & $47,8(45,8-49,7)$ & $21,3(20,8-21,9)$ \\
\hline $40-59$ & - & - & $57,6(55,3-59,9)$ & $17,2(16,6-17,8)$ \\
\hline 60 ou mais & - & - & $63,8(60,1-67,4)$ & $15,0(14,2-15,8)$ \\
\hline Brasil & $43,7(41,4-46,0)$ & $18,0(17,1-17,8)$ & $49,2(48,0-59,3)$ & $20,5(20,2-20,8)$ \\
\hline
\end{tabular}


quinto de participação calórica de alimentos ultraprocessados contra 84,1\% no quinto de maior participação). As hortaliças com maior participação na aquisição domiciliar foram: tomate $(29,2 \%$ do total de hortaliças adquiridas), cebola $(19,4 \%)$, cenoura $(8,1 \%)$, repolho (5,4\%), alface (4,8\%), abóbora (4,0\%), chuchu (3,4\%), pimentão (3,2\%), alho (3,1\%) e beterraba $(2,4 \%)$. Famílias com menor participação de ultraprocessados na alimentação apresentaram maior participação de repolho, abóbora e beterraba e menor de cebola, cenoura, chuchu e pimentão no total de hortaliças adquiridas (Tabela 3).

Em relação ao consumo alimentar individual, $42,1 \%$ dos brasileiros estudados não relataram consumo de hortaliças. Dentre aqueles que relataram ter consumido, a variedade ajustada de hortaliças foi de 1,08 tipo/dia para o conjunto da população. Indivíduos com menor consumo de alimentos ultraprocessados consumiram 1,14 tipo/dia de hortaliças e aqueles com maior consumo de ultraprocessados consumiram 1,05 tipo/dia ( $p=0,008)$ (dados não mostrados).

Os tipos de preparações mais consumidos pelos brasileiros foram hortaliças cruas (59,2\%), na forma cozida $(17,4 \%)$, como sopa $(15,4 \%)$ e refogadas $(6,8 \%)$. Entre os indivíduos com menor e maior consumo de alimentos ultraprocessados, a única diferença foi observada para a sopa, preparação mais consumida pelo grupo com menor consumo de alimentos ultraprocessados (Tabela 4).

Finalmente, verificou-se que o consumo de hortaliças se concentra em dois períodos do dia, equivalentes aos horários de almoço e jantar. No entanto, a quantidade consumida entre 11 horas e 14 horas é o dobro da quantidade consumida entre 19 horas e 22 horas. Indivíduos com menor consumo de alimentos ultraprocessados apresentam um menor consumo de hortaliças entre às 13 horas e 14 horas $(\mathrm{p}<0,05)$ e maior entre às 18 horas e 20 horas $(\mathrm{p}<0,001)$, quando comparados àqueles com maior consumo de ultraprocessados (Figura).

Tabela 2. Quantidade (em grama) de hortaliças adquiridas e consumidas no Brasil e segundo quintos da participação calórica de alimentos ultraprocessados: valores brutos e ajustados. Brasil, 2008-2009.

\begin{tabular}{|c|c|c|c|c|c|c|}
\hline $\begin{array}{l}\text { Quantidade de hortaliças } \\
\text { (g/per capita/dia) }\end{array}$ & Brasil & \multicolumn{5}{|c|}{$\begin{array}{l}\text { Quintos de participação calórica de alimentos ultraprocessados } \\
\text { (\% do total de energia) }\end{array}$} \\
\hline Aquisição domiciliar & & $\begin{array}{c}1^{\circ} \\
(9,0)\end{array}$ & $\begin{array}{c}2^{\circ} \\
(14,4)\end{array}$ & $\begin{array}{c}3^{\circ} \\
(17,4)\end{array}$ & $\begin{array}{c}4^{\circ} \\
(21,5)\end{array}$ & $\begin{array}{c}5^{\circ} \\
(27,4)\end{array}$ \\
\hline Valores brutos & 43,7 & 37,1 & 38,7 & 43,7 & 43,3 & $56,0^{c}$ \\
\hline Valores ajustados ${ }^{\mathrm{a}}$ & 42,9 & 46,9 & 44,4 & 41,9 & 39,4 & $36,9^{c}$ \\
\hline Consumo individual & & $\begin{array}{c}1^{\circ} \\
(1,8)\end{array}$ & $\begin{array}{l}2^{\circ} \\
(9,6)\end{array}$ & $\begin{array}{c}3^{\circ} \\
(17,9)\end{array}$ & $\begin{array}{c}4^{\circ} \\
(28,8)\end{array}$ & $\begin{array}{c}5^{\circ} \\
(49,2)\end{array}$ \\
\hline Valores brutos & 49,2 & 42,5 & 53,2 & 55,8 & 53,2 & 41,2 \\
\hline Valores ajustados ${ }^{\mathrm{b}}$ & 46,1 & 49,4 & 47,7 & 45,9 & 44,1 & $42,3^{\mathrm{c}}$ \\
\hline
\end{tabular}

a Modelo ajustado para renda per capita (em log), área e região.

${ }^{\mathrm{b}}$ Modelo ajustado para renda per capita (em log), área, região, sexo e idade.

${ }^{c} \mathrm{p}<0,05$ para tendência linear, obtido por modelo de regressão linear múltipla.

Tabela 3. Participação das hortaliças na aquisição domiciliar no Brasil e pelos brasileiros com menor e maior participação de alimentos ultraprocessados na alimentação. Brasil, 2008-2009.

\begin{tabular}{lccc}
\hline \multirow{2}{*}{ Hortaliças } & \multicolumn{2}{c}{ Participação (\%) na quantidade (grama) total de hortaliças adquiridas para consumo nos domicílios ${ }^{\mathrm{a}}$} \\
\cline { 2 - 4 } & Brasil & $\begin{array}{c}\mathbf{1}^{\mathbf{0}} \text { quinto de participação calórica de } \\
\text { alimentos ultraprocessados }\end{array}$ & $\begin{array}{c}\mathbf{5}^{\mathbf{0}} \text { quinto de participação calórica de } \\
\text { alimentos ultraprocessados }\end{array}$ \\
\hline Tomate & 29,2 & 28,9 & 30,7 \\
Cebola & 19,4 & 19,2 & $22,1^{\mathrm{b}}$ \\
Cenoura & 8,1 & 6,3 & $8,8^{\mathrm{b}}$ \\
Repolho & 5,4 & 6,5 & $3,9^{\mathrm{b}}$ \\
Alface & 4,8 & 4,2 & 4,3 \\
Abóbora & 4,0 & 5,9 & $2,6^{\mathrm{b}}$ \\
Chuchu & 3,4 & 3,0 & $3,8^{\mathrm{b}}$ \\
Pimentão & 3,2 & 2,7 & $3,5^{\mathrm{b}}$ \\
Alho & 3,1 & 3,3 & 2,6 \\
Beterraba & 2,4 & 2,8 & $1,9^{\mathrm{b}}$ \\
\hline Total & 82,9 & 82,7 & 84,1 \\
\hline
\end{tabular}

a Valores ajustados para renda per capita, área e região.

${ }^{\mathrm{b}} \mathrm{p}<0,05$ da tendência linear entre os quintos de consumo de alimentos ultraprocessados, obtido por modelo de regressão linear múltipla. 
Tabela 4. Tipos de preparações à base de hortaliças consumidas para o conjunto de brasileiros e entre aqueles com menor e maior consumo de alimentos ultraprocessados. Brasil, 2008-2009.

\begin{tabular}{lccc}
\hline & \multicolumn{3}{c}{ Participação (\%) na quantidade total de hortaliças consumidas ${ }^{\mathbf{a}}$} \\
\cline { 2 - 4 } Tipo de preparação & Brasil & $\begin{array}{r}\mathbf{1}^{\mathbf{0}} \text { quinto de participação calórica } \\
\text { de alimentos ultraprocessados }\end{array}$ & $\begin{array}{c}\mathbf{5}^{\mathbf{0}} \text { quinto de participação calórica } \\
\text { de alimentos ultraprocessados }\end{array}$ \\
\hline $\begin{array}{l}\text { Cru } \\
\text { Cozido }\end{array}$ & $\begin{array}{c}54,2 \\
\text { Sopa }\end{array}$ & 19,4 & 62,8 \\
$\begin{array}{l}\text { Refogado, alho e óleo } \\
\text { ou na manteiga }\end{array}$ & 15,4 & 18,6 & 16,6 \\
$\begin{array}{l}\text { Com molho ou } \\
\text { ensopado }\end{array}$ & 6,8 & 7,0 & $12,6^{\text {b }}$ \\
$\begin{array}{l}\text { Frito, empanado ou à } \\
\text { milanesa }\end{array}$ & 0,6 & 0,3 & 6,3 \\
$\begin{array}{l}\text { Grelhado, na brasa, } \\
\text { assado ou churrasco }\end{array}$ & 0,0 & 0,5 & 0,9 \\
\hline
\end{tabular}

${ }^{a}$ Valores ajustados para renda per capita (em log), área, região, sexo e idade.

${ }^{\mathrm{b}} \mathrm{p}<0,05$ da tendência linear entre os quintos de consumo de alimentos ultraprocessados, obtido por modelo de regressão linear múltipla.

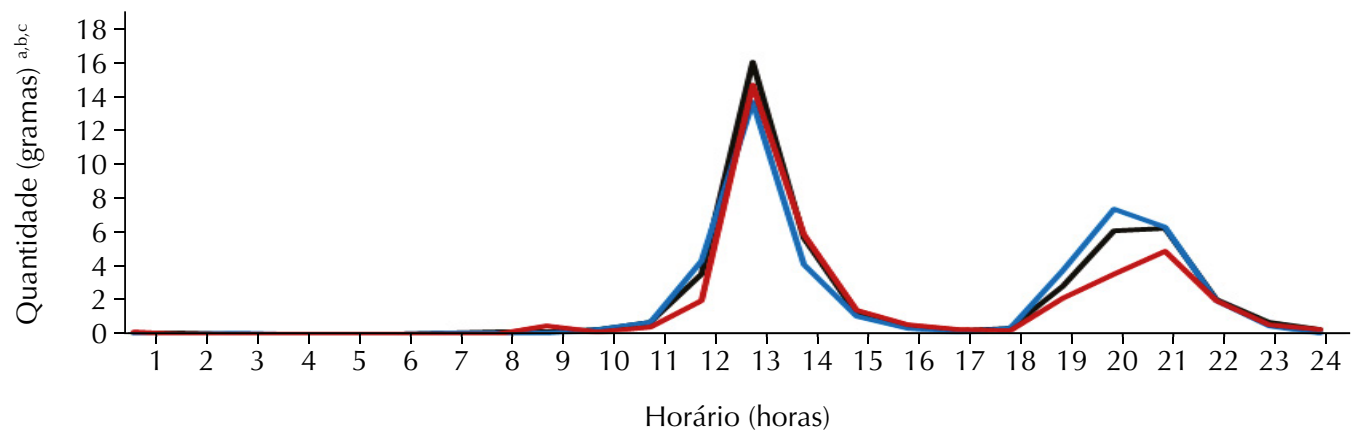

- Brasil

— $1^{\circ}$ quinto de participação de ultraprocessados

— $5^{\circ}$ quinto de participação de ultraprocessados

a Valores ajustados para renda per capita $(\log )$, área, região, sexo e idade.

${ }^{\mathrm{b}} \mathrm{p}<0,05$ (da tendência linear entre os quintos de consumo de alimentos ultraprocessados, obtido por modelo de regressão linear múltipla) para os seguintes horários: $8 \mathrm{~h}, 9 \mathrm{~h}, 14 \mathrm{~h}$.

c $p<0,001$ (da tendência linear entre os quintos de consumo de alimentos ultraprocessados, obtido por modelo de regressão linear múltipla) para os seguintes horários: $11 \mathrm{~h}, 13 \mathrm{~h}, 18 \mathrm{~h}, 19 \mathrm{~h}, 20 \mathrm{~h}$.

Figura. Quantidade de hortaliças consumidas (em grama), segundo horário de consumo, para o conjunto de brasileiros e entre aqueles com menor ( $1^{\circ}$ quinto de participação calórica) e maior ( $5^{\circ}$ quinto de participação calórica) consumo de alimentos ultraprocessados. Brasil, 2008-2009.

\section{DISCUSSÃO}

A análise dos dados de aquisição de alimentos para consumo domiciliar e de consumo alimentar individual da população brasileira, a partir dos dados da POF 2008-2009, evidenciou consumo insuficiente de hortaliças e sua relação inversa com o consumo de alimentos ultraprocessados. Observa-se que 10 tipos de hortaliças respondem por mais de $80 \%$ da quantidade total habitualmente adquirida. No consumo individual, verifica-se relativa monotonia, uma vez que a variedade predita de hortaliças foi de 1,08 tipo/dia. Cerca de $60 \%$ das hortaliças são consumidas cruas, sendo a quantidade consumida no almoço duas vezes maior do que aquela consumida no jantar. A quantidade de hortaliças consumida no almoço foi semelhante entre os diversos níveis de consumo de alimentos ultraprocessados; no jantar, indivíduos com menor consumo de alimentos ultraprocessados tenderam a consumir maior quantidade de hortaliças.

A relação inversa entre o consumo de hortaliças e de alimentos ultraprocessados reforça a já extensa relação de efeitos adversos relacionados ao consumo desses produtos. A partir disso, inferimos que, além dos malefícios ligados diretamente ao consumo dos ultraprocessados (em decorrência de seus atributos nutricionais negativos), esse consumo também prejudica indiretamente a 
k United States, Department of Agriculture, Economic Research Service. Selected charts from Ag and Food Statistics Charting the Essentials, 2017. Washington, DC: USDA; 2017.

'Ministério da Saúde (BR), Secretaria de Vigilância em Saúde. Vigitel Brasil 2014: vigilância de fatores de risco e proteção para doenças crônicas por inquérito telefônico. Brasília (DF); 2015 [citado 2 mar 2017]. Disponível em: http://svs.aids. gov.br/bases_vigitel_viva/

m Perez PMP. Impacto da implementação do Restaurante Universitário na alimentação de estudantes da Universidade do Estado do Rio de Janeiro [tese]. Rio de Janeiro: Universidade do Estado do Rio de Janeiro; 2015.

"Locatelli NT. Consumo da alimentação oferecida nas escolas públicas brasileiras e sua relação com fatores sociodemográficos e práticas alimentares: resultados da Pesquisa Nacional de Saúde do Escolar 2012 [dissertação]. Santos: Instituto de Saúde e Sociedade da Universidade Federal de São Paulo; 2016.

${ }^{\circ}$ Casa Civil (BR), Secretaria Especial de Agricultura Familiar e do Desenvolvimento Agrário. Programa de Aquisição de Alimentos. Brasília (DF); 2003 [citado 31 jan 2017]. Disponível em: http://www.mda.gov.br/ sitemda/secretaria/saf-paa/sobreo-programa alimentação, dada sua capacidade de deslocar e interferir no consumo de alimentos benéficos à saúde. Este resultado era esperado tendo em vista o corpo de evidência disponível quanto aos riscos do consumo de alimentos ultraprocessados ${ }^{15-18}$. Estudos avaliando o impacto dos alimentos ultraprocessados no perfil nutricional da alimentação dos brasileiros identificaram piora na qualidade da alimentação (maior densidade energética; maior quantidade de açúcar livre, gorduras saturadas e trans; e menor densidade de fibras, quantidade de proteínas e aporte de micronutrientes) com o aumento da participação de alimentos ultraprocessados na dieta ${ }^{11,12}$.

A despeito da enorme variedade de hortaliças encontradas no Brasil ${ }^{a}$, observou-se neste estudo uma limitada variação, uma vez que 10 tipos de hortaliças responderam por $83 \%$ da quantidade habitualmente consumida. Em relação ao número de hortaliças consumidas por dia, esse foi pouco maior que um em todas as situações analisadas, reforçando a baixa variedade de consumo. Apesar da importância da variedade de consumo para a qualidade da alimentação, esta temática tem sido pouco explorada na literatura ${ }^{14, k}$, não tendo sido localizado nenhum estudo brasileiro que descreva o consumo dos diferentes tipos de hortaliças ou mesmo intervenções com foco no incremento da variedade no consumo de hortaliças.

Em relação ao preparo, apesar da versatilidade das hortaliças ${ }^{1, a}$, em nosso estudo, mais da metade da quantidade desses alimentos foi consumida na forma crua. Ainda, estudos que descrevem os diferentes tipos de preparações consumidas são escassos, representando uma importante lacuna na literatura. Inquérito nacional conduzido com adolescentes encontrou que $27 \%$ desses jovens apresentavam um consumo regular de hortaliças cruas e $14 \%$, cozidas; entretanto, outros tipos de preparação não foram avaliados ${ }^{c}$. Por outro lado, a forma de preparo de hortaliças vem sendo abordada em estudos que avaliam o impacto de diferentes técnicas de preparo na composição nutricional ${ }^{19}$.

O maior consumo de hortaliças aconteceu durante o período do almoço (entre 12 horas e 14 horas), diferente do verificado nas populações norte-americana e norueguesa, que consomem estes alimentos principalmente no horário do jantar ${ }^{6,20}$. Esse resultado reflete a alta prevalência de consumo de refeições completas durante o almoço no país, sendo o jantar a refeição preferencialmente substituída por lanches. Dados do Vigitel, obtidos de uma amostra de indivíduos adultos ( $\geq 18$ anos) residindo nas 26 capitais de estado e Distrito Federal em 2014, indicam que, enquanto $16,5 \%$ da população estudada referia substituir o jantar por lanches (refeições com grande participação de alimentos ultraprocessados), apenas 1,4\% referiam fazê-lo no caso do almoçol. A maior substituição de refeição tradicional por lanche na hora do jantar explica, ainda que parcialmente, a razão pela qual os indivíduos que consomem mais alimentos ultraprocessados apresentam um menor consumo de hortaliças no período da noite.

Considerando os achados de nosso estudo, são necessárias estratégias de abordagem individual, coletiva e estruturante para melhora da qualidade da alimentação dos brasileiros. Como estratégia individual, destaca-se a necessidade de inclusão de hortaliças no jantar, em substituição a alimentos ultraprocessados ${ }^{20}$. Ademais, as intervenções devem focar na diversificação de hortaliças e das formas de preparo empregadas. Essas estratégias estão intimamente relacionadas às recomendações do Guia Alimentar, que aponta a importância da variedade da alimentação e do desenvolvimento de habilidades culinárias na promoção da alimentação saudável ${ }^{1}$.

Do ponto de vista coletivo, considerando a prática cada vez mais frequente de alimentação fora do lar, os cenários de alimentação coletiva, com destaque para o ambiente organizacional, podem ser considerados estratégicos para garantir a oferta e favorecer o consumo de hortaliças, como tem sido observado em alguns locais de trabalho, universidades e escolas ${ }^{21, m, n}$.

Ainda, estratégias estruturantes relacionadas à produção e distribuição de hortaliças, como o Programa de Aquisição de Alimentos ${ }^{0}$, são relevantes, uma vez que existem importantes disparidades no acesso a esses alimentos ${ }^{22-24}$. Além disso, nosso estudo não objetivou identificar fatores associados ao consumo de hortaliças no Brasil, mas questões relacionadas à falta de acesso físico ou financeiro a esses alimentos são frequentemente elencadas como determinantes centrais de seu baixo consumo ${ }^{25,26}$. 
Merece menção a opção de não analisar frutas e hortaliças em conjunto. Tomamos essa decisão porque esses alimentos, ainda que tratados em conjunto em recomendações nutricionais, tendem a diferir em relação à quantidade consumida ${ }^{9} \mathrm{e}$ às barreiras referidas para incremento do consumo ${ }^{27}$. Nesse sentido, considerando que intervenções com foco no maior consumo de frutas e de hortaliças necessitam de diferentes olhares, justifica-se a realização de análises mais detalhadas de cada um desses grupos.

O uso de registros alimentares, como os utilizados neste estudo, apresenta vieses potenciais, a saber: subestimação do consumo alimentar, alteração do consumo habitual nos dias de preenchimento do registro e diferenças entre as receitas das preparações culinárias praticadas pelos indivíduos e as receitas padronizadas utilizadas. A fim de minimizar esses vieses, o instrumento de coleta de dados empregado foi pré-testado e validado e foram empregados procedimentos de controle de qualidade durante a coleta de dados; além disso, registros inconsistentes foram excluídos e substituídos por valores imputados ${ }^{\mathrm{h}}$. Em relação à quantificação das hortaliças, potenciais erros foram minimizados com o emprego de padronização de sua participação nos diferentes tipos de preparações culinárias. Além disso, como o instrumento não foi desenvolvido para avaliar o consumo de alimentos segundo a extensão e o propósito de seu processamento industrial, alguns itens podem ter sido classificados incorretamente. Esses erros são mais prováveis para itens como pizzas, doces e sucos de frutas, que tanto podem ser classificados como preparações culinárias quanto como alimentos ultraprocessados. Em caso de ausência de informação nos registros que permitisse a distinção entre preparações culinárias e alimentos ultraprocessados (dado sobre a receita ou a marca do produto), optou-se por classificá-lo da forma mais frequente, considerando os casos em que havia informação.

Por outro lado, por utilizar dados de aquisição domiciliar e de consumo alimentar individual representativos da população brasileira, este estudo contribui de maneira importante para traçar um panorama, com diferentes olhares, sobre as práticas nacionais relacionadas ao consumo de hortaliças e sua relação com o consumo de alimentos ultraprocessados.

As diferenças de quantidade observadas para hortaliças adquiridas e consumidas podem ser referentes à técnica de preparo empregada, à incorporação de óleos e gorduras, a algum eventual desperdício de hortaliças ou ao consumo de alimentos fora do domicílio, mensurado apenas para o consumo individual. Além disso, os dados referentes à aquisição de alimentos permitem a identificação de hortaliças comumente utilizadas para temperar alimentos, como cebola e alho, as quais dificilmente aparecem de forma isolada em dados de consumo alimentar individual. Apesar de a literatura apontar similaridades entre dados de aquisição e consumo ${ }^{28-30}$, optou-se por apresentar as duas estimativas, uma vez que elas oferecem informações a respeito de dimensões distintas do consumo de hortaliças. Enquanto o dado de aquisição domiciliar expõe a intenção de consumo e a variedade habitual (livre de influência sazonal), o dado de consumo individual fornece informações a respeito da quantidade efetivamente ingerida, das preparações consumidas e do horário de consumo.

Conclui-se que o consumo de hortaliças no Brasil é insuficiente, sendo ainda menor entre indivíduos com maior consumo de alimentos ultraprocessados. O hábito mais frequente foi consumir hortaliças cruas, no horário do almoço e com limitada variedade.

\section{REFERÊNCIAS}

1. Ministério da Saúde (BR), Secretaria de Atenção à Saúde, Departamento de Atenção Básica. Guia alimentar para a população brasileira. 2.ed. Brasília (DF); 2014 [citado 2 mar 2017]. Disponível em: http://bvsms.saude.gov.br/bvs/publicacoes/guia_alimentar_populacao_ brasileira_2ed.pdf

2. Wang X, Ouyang Y, Liu J, Zhu M, Zhao G, Bao W, et al. Fruit and vegetable consumption and mortality from all causes, cardiovascular disease, and cancer: systematic review and dose-response meta-analysis of prospective cohort studies. BMJ. 2014;349:g4490. https://doi.org/10.1136/bmj.g4490 
3. Rezende LFM, Azeredo CM, Canella DS, Luiz OC, Levy RB, Eluf Neto J. Coronary heart disease mortality, cardiovascular disease mortality and all-cause mortality attributable to dietary intake over 20 years in Brazil. Int J Cardiol. 2016;217:64-8. https://doi.org/10.1016/j.ijcard.2016.04.176

4. World Health Organization. Diet, nutrition and the prevention of chronic diseases. Geneva: WHO; 2002. (WHO Technical Report Series, 916).

5. Australian Government, National Health and Medical Research Council. Australian dietary guidelines. Canberra: NHMRC; 2013.

6. Satia J, Kristal A, Patterson R, Neuhouser ML, Trudeau E. Psychosocial factors and dietary habits associated with vegetable intake. Nutrition. 2002;18(3):247-54. https://doi.org/10.1016/S0899-9007(01)00758-4

7. Abe SK, Stickley A, Roberts B, Richardson E, Abbott P, Rotman D, et al. Changing patterns of fruit and vegetable intake in countries of the former Soviet Union. Public Health Nutr. 2013;16(11):1924-32. https://doi.org/10.1017/S1368980013001316

8. Micha R, Khatibzadeh S, Shi P, Andrews KG, Engell RE, Mozaffarian D. Global, regional and national consumption of major food groups in 1990 and 2010: a systematic analysis including 266 country-specific nutrition surveys worldwide. BMJ Open. 2015;5(9):e008705. https://doi.org/10.1136/bmjopen-2015-008705

9. Martins APB, Levy RB, Claro RM, Moubarac J, Monteiro CA. Participação crescente de produtos ultraprocessados na dieta brasileira (1987-2009). Rev Saude Publica. 2013;47(4):656-65. https://doi.org/10.1590/S0034-8910.2013047004968

10. Azeredo CM, Rezende LFM, Canella DS, Claro RM, Castro IRR, Luiz OC, et al. Dietary intake of Brazilian adolescents. Public Health Nutr. 2015;18(7):1215-24. https://doi.org/10.1017/S1368980014001463

11. Louzada MLC, Martins APB, Canella DS, Baraldi LG, Levy RB, Claro RM, et al. Alimentos ultraprocessados e perfil nutricional da dieta no Brasil (2008-2009). Rev Saude Publica. 2015;49:38. https://doi.org/10.1590/S0034-8910.2015049006132

12. Louzada MLC, Martins APB, Canella DS, Baraldi LG, Levy RB, Claro RM, et al. Impacto de alimentos ultraprocessados sobre o teor de micronutrientes da dieta no Brasil. Rev Saude Publica. 2015;49:45. https://doi.org/10.1590/S0034-8910.2015049006211

13. Monteiro CA, Cannon G, Moubarac JC, Levy RB, Louzada MLC, Jaime PC. The UN Decade of Nutrition, the NOVA food classification and the trouble with ultra-processing. Public Health Nutr. 2018;21(1):5-17. https://doi.org/https://doi.org/10.1017/S1368980017000234

14. Oude Griep LM, Verschuren WM, Kromhout D, Ocké MC, Geleijnse JM. Variety in fruit and vegetable consumption and 10-year incidence of $\mathrm{CHD}$ and stroke. Public Health Nutr. 2012;15(12):2280-6. https://doi.org/10.1017/S1368980012000912

15. Canella DS, Levy RB, Martins APB, Claro RM, Moubarac JC, Baraldi LG, et al. Ultra-processed food products and obesity in Brazilian households (2008-2009). PLoS One. 2014;9(3):e92752. https://doi.org/10.1371/journal.pone.0092752

16. Rauber F, Campagnolo PD, Hoffman DJ, Vitolo MR. Consumption of ultra-processed food products and its effects on children's lipid profiles: a longitudinal study. Nutr Metab CardiovasC Dis. 2015;25(1):116-22. https://doi.org/10.1016/j.numecd.2014.08.001

17. Mendonça RD, Lopes ACS, Pimenta AM, Gea A, Martinez-Gonzalez MA, Bes-Rastrollo M. Ultra-processed food consumption and the incidence of hypertension in a Mediterranean cohort: The Seguimiento Universidad de Navarra Project. Am J Hypertens. 2017;30(4):358-66. https://doi.org/https://doi.org/10.1093/ajh/hpw137

18. Mendonça RD, Pimenta AM, Gea A, Fuente-Arrillaga C, Martinez-Gonzalez MA, Lopes ACS, et al. Ultra-processed foods consumption and risk of overweight and obesity: The University of Navarra Follow-Up (SUN) cohort study. Am J Clin Nutr. 2016;104(5):1433-40. https://doi.org/10.3945/ajcn.116.135004

19. Murador DC, Cunha DT, Rosso VV. Effects of cooking techniques on vegetable pigments: a metaanalytic approach to carotenoid and anthocyanin levels. Food Res Int. 2014;65(Pt B):177-83. https://doi.org/10.1016/j.foodres.2014.06.015

20. Myhre JB, Løken EB, Wandel M, Andersen LF. Meal types as sources for intakes of fruits, vegetables, fish and whole grains among Norwegian adults. Public Health Nutr. 2015;18(11):2011-21. https://doi.org/10.1017/S1368980014002481

21. Bandoni DH, Sarno F, Jaime PC. Impact of an intervention on the availability and consumption of fruits and vegetables in the workplace. Public Health Nutr. 2011;14(6):975-81. https://doi.org/10.1017/S1368980010003460 
22. Jaime PC, Machado FMS, Westphal MF, Monteiro CA. Impact of a community-based intervention to increase fruit and vegetable intake among low-income families from São Paulo, Brasil. Rev Chil Nutr. 2006;33 Supl 1:266-71. https://doi.org/10.4067/S0717-75182006000300008

23. Duran AC, Diez-Roux AV, Latorre MRDO, Jaime PC. Neighborhood socioeconomic characteristics and differences in the availability of healthy food stores and restaurants in Sao Paulo, Brazil. Health Place. 2013;23:39-47. https://doi.org/10.1016/j.healthplace.2013.05.001

24. Pessoa MC, Mendes LL, Gomes CS, Martins PA, Velasquez-Melendez G. Food environment and fruit and vegetable intake in a urban population: a multilevel analysis. BMC Public Health. 2015;15:1012. https://doi.org/10.1186/s12889-015-2277-1

25. Claro RM, Monteiro CA. Renda familiar, preço de alimentos e aquisição domiciliar de frutas e hortaliças no Brasil. Rev Saude Publica. 2010;44(6):1014-20. https://doi.org/10.1590/S0034-89102010000600005

26. Chor D, Cardoso LO, Nobre AA, Griep RH, Fonseca MJM, Giatti L, et al. Association between perceived neighbourhood characteristics, physical activity and diet quality: results of the Brazilian Longitudinal Study of Adult Health (ELSA-Brasil). BMC Public Health. 2016;16:751. https://doi.org/10.1186/s12889-016-3447-5

27. Glasson C, Chapman K, James E. Fruit and vegetables should be targeted separately in health promotion programmes: differences in consumption levels, barriers, knowledge and stages of readiness for change. Public Health Nutr. 2011;14(4):694-701. https://doi.org/10.1017/S1368980010001643

28. Becker W. Comparability of household and individual food consumption data: evidence from Sweden. Public Health Nutr. 2001;4(5B):1177-82. https://doi.org/10.1079/PHN2001197

29. Naska A, Vasdekis VGS, Trichopoulou A. A preliminary assessment of the use of household budget survey data for the prediction of individual food consumption. Public Health Nutr. 2001;4(5B):1159-65. https://doi.org/10.1079/PHN2001237

30. Claro RM, Jaime PC, Lock K, Fisberg RM, Monteiro CA. Discrepancies among ecological, household, and individual data on fruits and vegetables consumption in Brazil. Cad Saude Publica. 2010;26(11):2168-76. https://doi.org/10.1590/S0102-311X2010001100018

Financiamento: Conselho Nacional de Desenvolvimento Científico e Tecnológico (CNPq - Apoio a projeto, Chamada Universal MCTI/CNPQ 14/2014 - Processo 457801/2014-0).

Contribuição dos Autores: Concepção e planejamento do estudo: DSC, MLCL, APBM. Análise dos dados: DSC, MLCL. Interpretação dos dados: DSC, MLCL, RMC, JCC, DHB, RBL. Redação do manuscrito: DSC, JCC. Revisão crítica do manuscrito: MLCL, RMC, DHB, RBL, APBM. Aprovação da versão final: DSC, MLCL, RMC, JCC, DHB, RBL, APBM.

Conflito de Interesses: Os autores declaram não haver conflito de interesses. 